\title{
Magnetic field distribution simulation and performance experiment of a magnetic seed-metering device based on the combined magnetic system
}

\author{
Wei Liu, Jianping $\mathrm{Hu}^{*}$, Mengjiao Yao, Jun Zhao, Imran Ali Lakhiar, Chuantong Lu, \\ Haoran Pan, Wei Wang \\ (School of Agricultural Equipment Engineering, Jiangsu University, Zhenjiang 212013, Jiangsu, China)
}

\begin{abstract}
The magnetic seed-metering method is one of the universal approaches for plug tray seeding. Conventional electromagnetic and permanent-magnetic seed-metering devices either cannot supply a desirable magnitude of magnetic force or need a seed-clearing mechanism. Thus the objective of this research was to develop a combined magnetic system (CMS) seed-metering device for generating higher magnetic forces than the electromagnetic ones without using any auxiliary seed-clearing mechanisms. Firstly, the CMS component was designed and its magnetic field distributions in both attractive and clearing states were obtained by finite element method simulations. Secondly, based on the CMS components, a magnetic seed-metering device was developed and validated by a prototype experiment. The simulation results displayed that along the axial direction of the magnetic head, the maximum magnetic flux density in the clearing state was $21.03 \%$ of that in the attractive state. In addition, along the radial direction, the proportion was $24.16 \%$. Concerning the spatial magnetic flux density distribution, the magnetic flux density on the seeding planes of CMS components (approximately $60 \mathrm{mT}$ ) was higher than that of transitional space between two CMS components (nearly $0 \mathrm{mT}$ ). As for the seeding performance experiment, when the rotational speed of the roller was $21 \mathrm{r} / \mathrm{min}$ and the exciting current was $0.15 \mathrm{~A}$, the highest single rate was acquired $(90.20 \%)$. In the same condition, the reseeding rate was $5.88 \%$ and the miss-seeding rate was $3.92 \%$. The results suggest that the magnetic field distribution and seeding performance of the developed magnetic seed-metering device are acceptable. Therefore, the developed magnetic seed-metering device can be used in practical plug tray seeding processes.
\end{abstract}

Keywords: seed-metering device, magnetic field, magnetic flux density, finite element method, simulation DOI: $10.25165 /$ j.ijabe.20211401.5852

Citation: Liu W, Hu J P, Yao M J, Zhao J, Lakhiar I A, Lu C T, et al. Magnetic field distribution simulation and performance experiment of a magnetic seed-metering device based on the combined magnetic system. Int J Agric \& Biol Eng, 2021; 14(1): $108-117$

\section{Introduction}

At present, an increasing number of high-value-added vegetables, such as cucumber, tomato, and capsicum are planted in plant factories ${ }^{[1]}$. In order to adapt to the cultivation mode of the plant factory, seeds need to be sown in plug trays. The seeding process of these vegetables is implemented by precision plug tray seeders. Unlike precision seeders used in the field, precision plug tray seeders sow vegetable seeds into plug trays, rather than discharging them into the field directly ${ }^{[2,3]}$. The seed-metering device is the core component of a precision plug tray seeder, and the performance of the seed-metering device is crucial to seeding

Received date: 2020-04-16 Accepted date: 2020-08-27

Biographies: Wei Liu, PhD candidate, research interest: intelligent measurement and controlling for seeding machinery, Email: lw19900714@163.com; Mengjiao Yao, MS candidate, research interest: intelligent seeding machine, Email: ymj_research@163.com; Jun Zhao, MS candidate, research interest: seeding machinery design, Email: zj145995@163.com; Imran Ali Lakhiar, $\mathrm{PhD}$, research interest: soil-water engineering, aeroponics and hydroponics systems, Email: 5103160321@stmail.ujs.edu.cn; Chuantong Lu, MS, research interest: seeding machinery design, Email: luchuantong1989@sina.com; Haoran Pan, MS candidate, research interest: seeding machinery design, Email: michaelzhipan@foxmail.com; Wei Wang, PhD candidate, research interest: seeding machinery design, Email: 17826077597@163.com.

*Corresponding author: Jianping Hu, Professor, research interest: seeding and planting machinery. School of Agricultural Equipment Engineering, Jiangsu University, Zhenjiang 212013, Jiangsu, China. Tel: +86-13852984643, Email: hujp@ujs.edu.cn. quality and efficiency.

Studies reported that the mechanical, pneumatic and magnetic seed-metering devices are the most common apparatuses for plug tray seeding ${ }^{[4-6]}$. As for a mechanical seed-metering device, seeds' filling, holding and discharging processes are completed by the seeds' gravity and mechanical device ${ }^{[7-9]}$. Although the structure of the mechanical seed-metering device is simple, it is designed strictly in accordance with the seeds' shape and volume, limiting its versatility ${ }^{[10]}$. Another shortcoming of the device is that seeds are prone to be damaged in seeding processes due to physical contacts ${ }^{[1,12]}$.

Moreover, the pneumatic seed-metering device gradually replaces the mechanical version, because of less seed damage. Sown by the pneumatic seed-metering devices, seeds are held at outlets of nozzles by negative pressure, which reduces the contacts with mechanisms ${ }^{[13,14]}$. However, a pneumatic system would complicate the structure of the seed-metering device and nozzles need to be replaced before sowing seeds of different types. Furthermore, a study by Guarella et al. ${ }^{[15]}$ reported that if the sown seeds have a small volume, the diameter of nozzles must be smaller than the seeds' diameter because the nozzles could be blocked due to their small diameter.

In order to save time for replacing nozzles and avoid nozzle being blocked, $\mathrm{Hu}$ and $\mathrm{Mao}^{[16]}$ proposed a magnetic seeding theory and developed a magnetic seed-metering device. This study further reported that before seeding operations, a mixture of iron powder and coating one should be covered on seeds in a pelleting 
process. The magnetic seeding device used columnar electromagnets as seeding components to attract and hold the pelleted seeds. These columnar electromagnets were driven by a parallelogram reciprocating mechanism to move from a hopper to plug tray cells. The magnetic seed-metering method can adapt the seeds with different volumes by adjusting the magnitude of the exciting current, leading to saving the time for replacing nozzles and reducing the effect of seeds' irregular shapes. Another study by $\mathrm{Li}^{[17]}$ improved the kinetic stability of electromagnets and seeding efficiency by applying a roller to replace the parallelogram mechanism and optimized the structure parameters of the magnetic seeding component. Furthermore, the basic conditions of attractive, holding and discharging seeds were determined by the means of mechanical analyses. In 2010, Hu et al. ${ }^{[18]}$ developed a permanent-magnetic seed-metering device for improving the magnetic flux density of each seeding component. They used a cum mechanism to control the permanent-magnetic seeding components to reach out and retract the roller surface periodically, for discharging and attractive seeds. Lately, a plate-type magnetic seeding device, whose size was in accordance with that of a universal plug tray, was developed by Yan et al. ${ }^{[19]}$. In practical operations, the plate-type magnetic seeding device would flip over the plug tray and then discharge all pelleted seeds into plug tray cells at the same time. Although the types of magnetic seed-metering devices are various, the basic theories can only be categorized into two types, namely, the electromagnetic method and the permanent-magnetic one. Concerning the electromagnetic approach, its magnetic flux density can be easily adjusted by the exciting current, but the magnitude of magnetic flux density is limited ${ }^{[20]}$. Also, it would generate a great deal of heat in working conditions, which leads to reducing magnetic force and usage time of coils. As for the permanent-magnetic seed-metering device, its magnetic flux density is larger than that generated by an electromagnetic seed-metering device. Nevertheless, the permanent-magnet seed-metering device needs an extra seed-clearing mechanism to clear seeds, which would make the structure of the seed-metering device complex ${ }^{[21]}$.

So as to overcome the aforementioned shortcomings of electromagnetic and permanent-magnetic seed-metering devices, the objective of this research was to develop a magnetic seed-metering device that not only can generate sufficient magnetic flux densities but also can avoid having a complex mechanical structure. In order to achieve the objects of the present study, we planned the work in different sections, and they were organized as follows:

(a) At first, a seeding component based on a combined magnetic system (CMS) was designed and its magnetic field distribution was obtained by finite element method (FEM) simulations.

(b) In the next step, a magnetic seed-metering device based on the proposed CMS components was developed. In addition, the spatial magnetic field of the developed magnetic seed-metering device was simulated to reveal whether there was an interaction between two adjacent CMS components.

(c) At last, the optimal working parameters were determined by a seeding performance experiment. Also, the effect of each factor on the seeding performance was obtained.

The motivations of sections (b) and (c) were based on the resultant analyses and inferences of their former sections. Therefore, the body of this paper was organized chronologically. The basis of this research was the development of the CMS component; therefore, it is demonstrated at the beginning of the body of this study.

\section{Development and simulation analyses of the CMS component}

\subsection{Structure and working principle of the CMS component}

The seeding components were taken as the most critical parts of a magnetic seed-metering device. A combined magnetic system (CMS) was put forward to improve the magnetic force of pelleted seeds. In addition, it did not need any auxiliary seed-clearing mechanisms, thereby reducing the complexity of the whole seed-metering device.

A CMS component consisted of two subsystems: a magnetic force supplying subsystem (MFSS) and a magnetic-circuit controlling subsystem (MCCS) (Figure 1a). The MFSS, which supplied the magnetic force for holding the pelleted seeds, was composed of two permanent-magnets and a magnetic head. The MCCS contained a coil and an iron core, and it was used to control the magnetic-circuit direction of the MFSS. The different magnetic-circuit directions can result in distinct magnetic flux density distributions on the seeding plane. Hence, the magnetic force applied on a pelleted seed was changed.

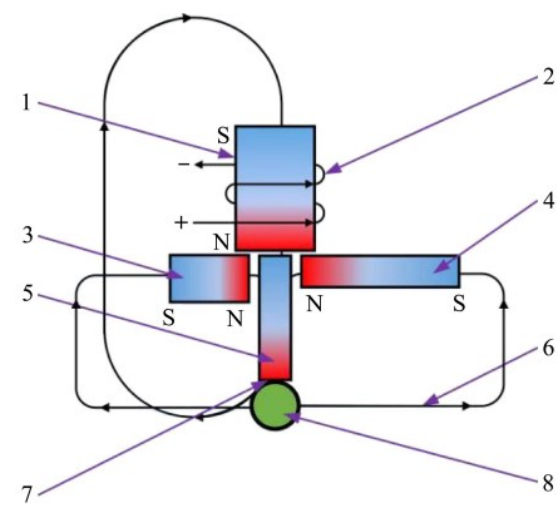

a. Attractive state

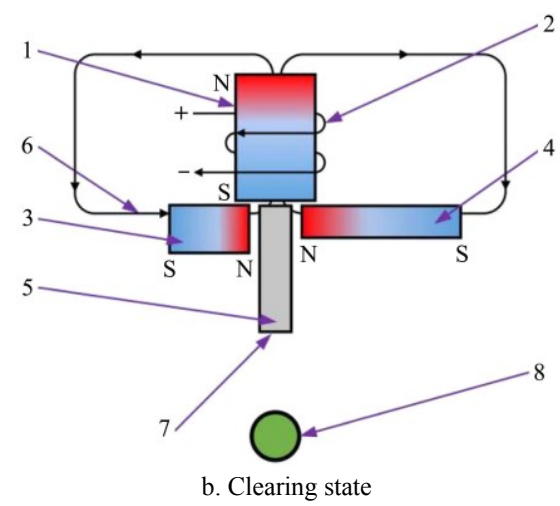

1. Iron core 2 Coil 3. Permanent-magnet I 4. Permanent-magnet II 5. Magnetic head 6. Magnetic induction line 7. Seeding plane 8. Pelleted seed.

Figure 1 Structure of CMS component and magnetic-circuit directions in two working states

The size of cylinder permanent magnets I and II were different (Figure 1). The diameter and length of the permanent magnet I were $3 \mathrm{~mm}$ and $5 \mathrm{~mm}$, while those of the permanent-magnet II were $2 \mathrm{~mm}$ and $10 \mathrm{~mm}$. These two permanent-magnets can generate a bias magnetic field which can result in an obvious magnetic flux density difference when switching the attractive state to the clearing one.

There were two working states of the CMS componentattractive and clearing. The magnetic-circuits of the CMS 
component in the attractive state are shown in Figure 1a. The iron core and the permanent-magnets had identical polarities at the top of the magnetic head ( $\mathrm{N}$ pole). The magnetic-circuits of the MCCS formed a closed-loop via the iron core, air gap, and magnetic head. The magnetic-circuits of the MFSS passed through the permanent-magnets, air gap and magnetic head. Because all magnetic-circuits of the two subsystems would flow through the magnetic head, the magnetic flux density at the seeding plane was reinforced. Pelleted seeds, therefore, can be picked up on the seeding plane.

If the exciting current was applied reversely, the working state of the CMS component would be switched to the clearing one whose magnetic-circuits are shown in Figure 1b. At the top part of the magnetic head, the polarity of the electromagnet (S pole) was different from those of the permanent-magnets $(\mathrm{N}$ pole). There were no magnetic induction lines via the seeding plane. The magnetic force generated by remanence was less than the pelleted seeds' gravity. Hence, the pelleted seeds would fall.

\subsection{Magnetic field simulation of the CMS component and} model validation

The magnetic field distribution of a CMS component determined whether a seed can be attracted to the seeding plane or not. The magnetic field distributions of the CMS component in the attractive and clearing states were simulated in electro-magnetic field FEM software Maxwell 12.0 (ANSYS Inc., Pittsburgh, PA, USA). In addition, the material and size of each part of the CMS component are listed in Table 1.

Table 1 Material and size of each part of the CMS component

\begin{tabular}{lcccc}
\hline \multicolumn{1}{c}{ Component } & Material & $\begin{array}{c}\text { Diameter } \\
/ \mathrm{mm}\end{array}$ & $\begin{array}{c}\text { Length } \\
/ \mathrm{mm}\end{array}$ & $\begin{array}{c}\text { Number of } \\
\text { Windings }\end{array}$ \\
\hline permanent-magnet 1 & NdFe35 & 3 & 5 & \\
permanent-magnet 2 & NdFe35 & 2 & 10 & - \\
Iron core & Permalloy 1J22 & 2 & 10 & \\
magnetic head & Permalloy 1J22 & 2 & 7 & 2000 \\
\hline Coil & Copper & 14 & 10 & 2000 \\
\hline
\end{tabular}

The solution type was magnetostatics type and the solver boundary was selected as the balloon boundary. As for mesh operations, taking the computational speed and precision into consideration, we selected the adaptively refined mesh method for element segmentation. As a result, the end parts of permanentmagnets and magnetic head were segmented densely; the meshes at other sections were relatively sparse (Figure 2). Besides, Table 2 lists the computational parameters set in setup tags.

The premise of obtaining credible simulation results was that the simulation model had sufficient precision. To validate the model precision, the actual magnetic flux densities need to be compared with the simulated ones. Thus, a teslameter (KANETEC TM710, Tokyo, Japan) was used to measure the magnetic flux densities at different distances from the seeding plane. The length and width of the detection plane of the teslameter were $2 \mathrm{~mm}$ and $1 \mathrm{~mm}$, respectively. Furthermore, the exciting current was applied as same as the simulation value (0.2 A). A CMS component was horizontally fixed on a base and measuring distances were selected from $0 \mathrm{~mm}$ to $5 \mathrm{~mm}$ averagely with an interval of $1 \mathrm{~mm}$.

In addition, the relative error was used to evaluate the model precision, which is illustrated in Equation (1):

$$
R E=\left(1-\left|\frac{B_{s}}{B_{a}}-1\right|\right) \times 100 \%
$$

where, $R E$ is the relative error, \%; $B_{a}$ stands for the measured magnetic flux density, $\mathrm{mT} ; B_{s}$ represents the simulation value of magnetic flux density, $\mathrm{mT}$.

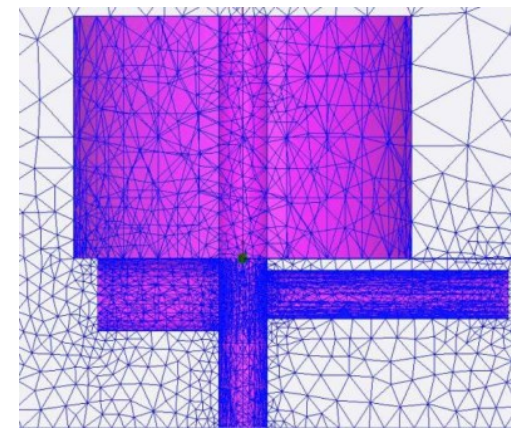

Figure 2 Computational grids of the CMS component

Table 2 Computational parameters set in the setup tags

\begin{tabular}{lc}
\hline \multicolumn{1}{c}{ Computational parameter } & Value \\
\hline The value of current excitation/A & 400 \\
The maximum number of passes & 10 \\
Percent error & 1 \\
Refinement per pass $\%$ & 30 \\
Minimum number of passes & 2 \\
Minimum converged passes & 1 \\
\hline
\end{tabular}

Note: The value of current excitation (400) in the software should be set as the product of the exciting current and the windings number of the coil $(0.2 \times 2000)$.

Table 3 shows the simulation and measured magnetic flux densities at the distances $0 \mathrm{~mm}$ to $5 \mathrm{~mm}$ away from the seeding plane. During the test, the magnetic flux density was the maximum when the measuring distance was $0 \mathrm{~mm}$ and the simulation and measured values were $151.27 \mathrm{mT}$ and $129.0 \mathrm{mT}$, respectively. In contrast, the value was the minimum when the distance was $5 \mathrm{~mm}$ and the simulation and measured values were $9.648 \mathrm{mT}$ and $10.0 \mathrm{mT}$, respectively. Besides, the magnetic flux density would decline gradually as the measuring distance increased.

Table 3 Simulation and measurement values at the distance from $0 \mathrm{~mm}$ to $5 \mathrm{~mm}$

\begin{tabular}{cccc}
\hline Distance/mm & Simulation value/mT & Measurement value/mT & RE/\% \\
\hline 0 & 151.274 & 129.0 & 14.7240 \\
1 & 84.721 & 80.9 & 4.5101 \\
2 & 40.840 & 39.1 & 4.2605 \\
3 & 23.385 & 22.4 & 4.2121 \\
4 & 14.640 & 14.2 & 3.0055 \\
5 & 9.648 & 10.0 & 3.6480 \\
\hline
\end{tabular}

As can be seen in Table 3, when the distance was $0 \mathrm{~mm}$, the highest relative error $(14.72 \%)$ was observed. In the simulation environment, the detection plane and the seeding plane overlapped completely, so the simulation value was the theoretical one at the center of the seeding plane. In practical measurement, an air gap between the detection plane and the seeding plane may be difficult to be eliminated, resulting in a measuring error.

Moreover, when the measuring distance was from $0 \mathrm{~mm}$ to $5 \mathrm{~mm}$, the average value of the relative errors was $5.73 \%$. According to the average relative error, it can be concluded that the precision of the simulation model of the CMS component is acceptable and subsequent simulation results are credible.

\subsection{Magnetic flux density comparison with the} electromagnetic component

If the max magnetic flux density of the CMS component was higher than that of an electromagnetic one, the less iron powder 
needed mixing in the coating powder. In addition, if the CMS component had a wider adjustable range of the magnetic flux density, it can adapt to more types of pelleted seeds of different volumes. In order to compare the magnetic flux densities of the conventional electromagnetic component, the magnetic flux densities of a CMS component were measured when applying different magnitudes of the exciting current.

The measurement method is described in Section 2.2. In the measurements, the exciting current was applied to the CMS component at 6 magnitudes, namely $0.05 \mathrm{~A}, 0.10 \mathrm{~A}, 0.15 \mathrm{~A}, 0.20 \mathrm{~A}$, $0.25 \mathrm{~A}$, and $0.30 \mathrm{~A}$, respectively. However, the measuring distances were averagely selected from $0 \mathrm{~mm}$ to $3 \mathrm{~mm}$ away from the seeding plane with an interval of $0.05 \mathrm{~mm}$.

Moreover, when the CMS component was applied to each exciting current, the measured magnetic flux densities of the seeding plane are shown in Figure 3. The distances measured from $0 \mathrm{~mm}$ to $3 \mathrm{~mm}$. The magnetic flux densities of an electromagnetic component applied the same exciting currents as previously reported by Zheng ${ }^{[22]}$.

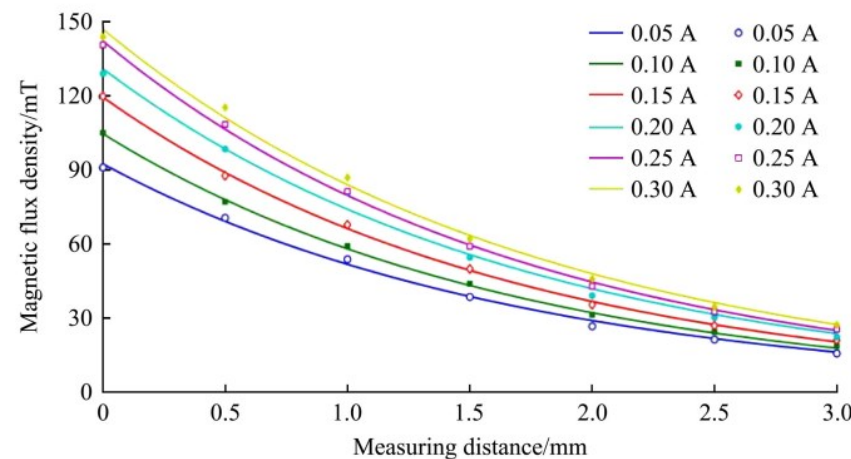

Figure 3 Relationship between measuring distances and magnetic flux densities of the CMS component applied different exciting currents

When exciting current was $0.30 \mathrm{~A}$, the max magnetic flux density of the CMS component and the electromagnetic component were $143.9 \mathrm{mT}$ and $68.2 \mathrm{mT}$, respectively. Under the same exciting current level, the CMS component can generate a higher magnetic flux density, compared to the electromagnetic component. Furthermore, when applying the CMS components instead of the electromagnetic ones, less iron powder is necessary to be used.

Owing to being covered by different volumes of iron powder, every type of pelleted seeds needed an appropriate magnetic flux density, which could result in the highest single rate. The CMS component had a wider adjustable range of the magnetic flux density on the seeding plane (i.e., the measuring distance was $0 \mathrm{~mm}$ ) compared to the electromagnetic one (52.8 $\mathrm{mT}$ versus $30.4 \mathrm{mT})$. Thus it could adapt more types of pelleted seeds. Hence, sowing different seeds need not replace CMS components but only to apply different exciting currents.

The essential reason why the magnetic flux density of the CMS seeding component was improved compared to that of an electromagnetic component could be explained as follows. The main magnetic force used for attracting seeds was supplied by the two permanent-magnets; the electromagnet was only used for controlling the direction of magnetic-circuits of permanent-magnets, like a switch. In contrast, the magnetic force of the conventional electromagnetic component was supplied by the electromagnet. Thus, by applying the same exciting current, the CMS component could generate a higher magnetic force compared to the electromagnetic one.
When the magnitude of the exciting current was a certain value, it was found that the magnetic flux density exponentially declined as the measuring distance increased. In addition, the relationship between magnetic flux density and measuring distance can be illustrated by Equation (2):

$$
B=a \cdot \exp (b \cdot D)
$$

where, $B$ represents the magnetic flux density, $\mathrm{mT} ; D$ is the measuring distance, $\mathrm{mm} ; a$ and $b$ are the coefficients of the fitting equation.

Under each exciting current level, the function of magnetic flux density and measuring distance was fitted with $95 \%$ confidence bounds. All functions were fitted using the curve fitting toolbox of Matlab 2017 (Mathworks Inc., Natick, MA, USA). Moreover, the coefficients and index of these fitted functions are listed in Table 4. The lines in Figure 3 are the fitted curves of the magnetic flux densities at different measuring distances.

Table 4 Coefficients and index of fitting functions of magnetic flux density and measuring distances

\begin{tabular}{cccccc}
\hline \multirow{2}{*}{$\begin{array}{c}\text { Exciting current } \\
\text { /A }\end{array}$} & \multicolumn{2}{c}{ Coefficient } & & \multicolumn{2}{c}{ Fitting index } \\
\cline { 2 - 5 } \cline { 4 - 5 } & $a$ & $B$ & & $R^{2}$ & RMSE \\
\hline 0.05 & 92.48 & -0.58 & 0.997 & 1.663 \\
0.10 & 104.70 & -0.59 & 0.993 & 0.876 \\
0.15 & 119.50 & -0.59 & 0.999 & 1.180 \\
0.20 & 131.00 & -0.57 & 0.993 & 3.540 \\
0.25 & 142.30 & -0.58 & 0.999 & 1.639 \\
0.30 & 147.10 & -0.56 & 0.996 & 3.015 \\
\hline
\end{tabular}

Note: $R^{2}$ is the coefficient of determination and RMSE represents the root mean squared error.

In Table 4 , it is found that parameter $a$ is proportional to the increasing magnitude of the exciting current. Thus, parameter $a$ can be represented by a one-order function of the exciting current. However, the parameter $b$ almost did not vary as the exciting current increased, so the average value of the parameter $b(-0.58)$ can represent the coefficient $b$ under all exciting current levels. Therefore, the parameter $a$ in Equation (2) can be replaced with the linear relationship of exciting current $i$, as Equation (3):

$$
B=(227.09 i+83.11) \cdot \exp (-0.58 D)
$$

The value range of exciting current $i$ is from $0.05 \mathrm{~A}$ to $0.30 \mathrm{~A}$. Therefore, Equation (3) can be used to compute the theoretical magnetic flux densities along the axis direction of the magnetic head.

\subsection{Simulation results of the magnetic flux density} distribution of the CMS component and resultant analyses

Figure $4 \mathrm{a}$ shows that the magnetic flux density distribution of a CMS component working in an attractive state. However, Figure $4 \mathrm{~b}$ indicates the magnetic flux density distribution on the seeding plane.

As shown in Figure 4a, the area of highest magnetic flux density is mainly concentrated around the magnetic head, permanent-magnet I and two ends of permanent-magnet II. Magnetic induction lines would intensively come out from the $\mathrm{N}$ poles of two permanent-magnets and return into their $\mathrm{S}$ poles (Figure 1a). Accordingly, the magnetic flux density was relatively high at the ends of these two permanent-magnets. As for the magnetic head, it worked as a magnet yoke in the CMS component. The magnetic induction lines of both the MCCS and the MFSS would merge, and pass through the magnetic head. Consequently, the surrounding space of the magnetic head had a 
relatively high magnetic flux density.

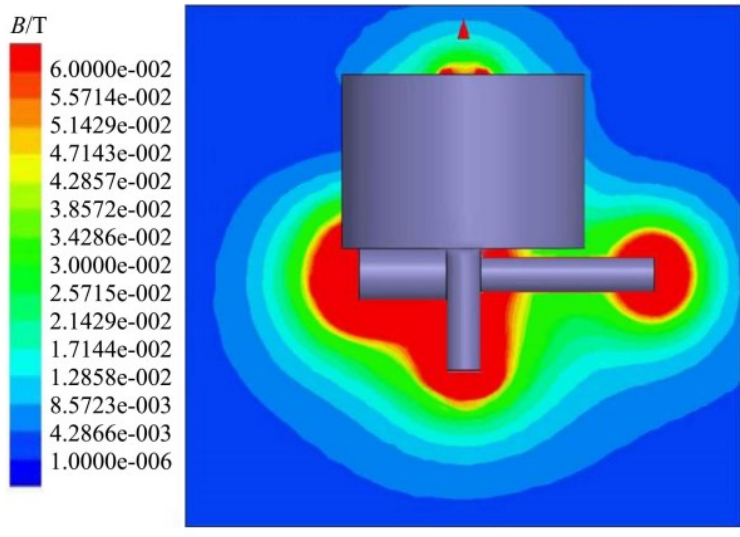

a. Around the CMS component

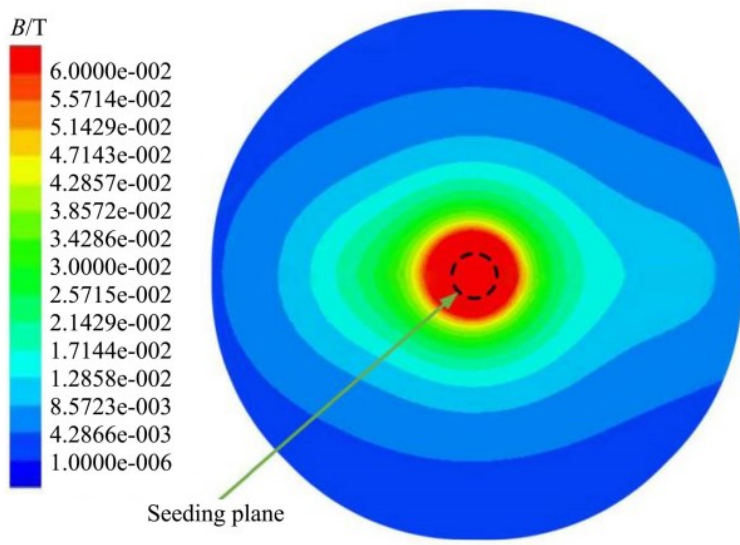

b. On the seeding plane

Figure 4 Magnetic flux density distribution of the CMS component working in the attractive state

Moreover, the magnetic flux density on the side of the permanent-magnet I was larger than that around the permanent-magnet II. Magnetic induction lines of the permanent-magnet I had a small irradiation loop than that of the permanent-magnet II due to its smaller length and larger end face area.

Figure 5a shows the magnetic flux density distribution when the CMS component works in the clearing state. The high area of magnetic flux density is concentrated on the left of permanent-magnet I, right of the permanent-magnet II and the top of the iron core.

When the working state of the CMS component was switched from the attractive state to the clearing one, the polarities of the ends of the iron core would exchange, thereby transforming the magnetic-circuit direction of the MFSS. The magnetic induction lines flowed out of the $\mathrm{N}$ poles of two permanent-magnets and flowed back to their S poles, through the iron core, as seen in Figure 1b. Since the remanence of the magnetic head was weak, rare sections around the magnetic head were marked in red in Figure 5a.

Figure $5 \mathrm{~b}$ illustrates the nephogram of magnetic flux density on the seeding plane when the CMS component works in the clearing state. Relatively high magnetic flux density concentrated on the perimeter of the seeding plane. It suggests that the edge of the magnetic head may have an effect on magnetism gathering.

Figure 6a shows the magnitudes of magnetic flux density along the center axis of the magnetic head. The maximum magnetic flux density was $148.58 \mathrm{mT}$ and it fell sharply as the distance increased from $0 \mathrm{~mm}$ to $3 \mathrm{~mm}$. However, the maximum of magnetic flux densities in the clearing state was $31.25 \mathrm{mT}$ which was $21.03 \%$ of that in the attractive state.
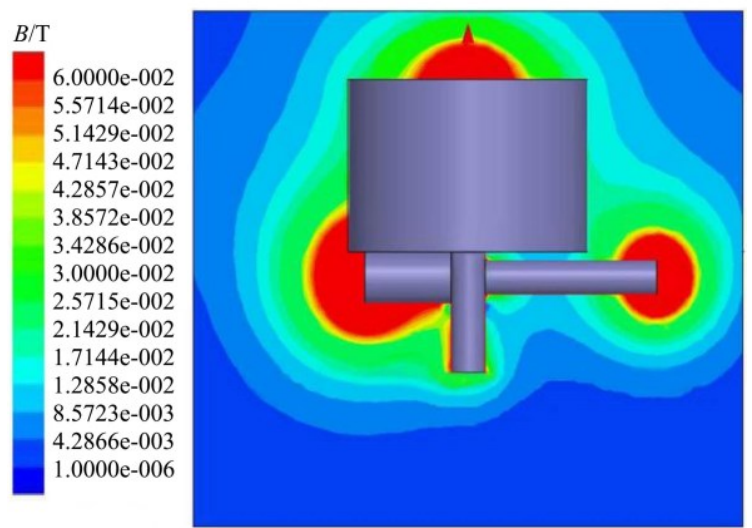

a. Around the CMS component

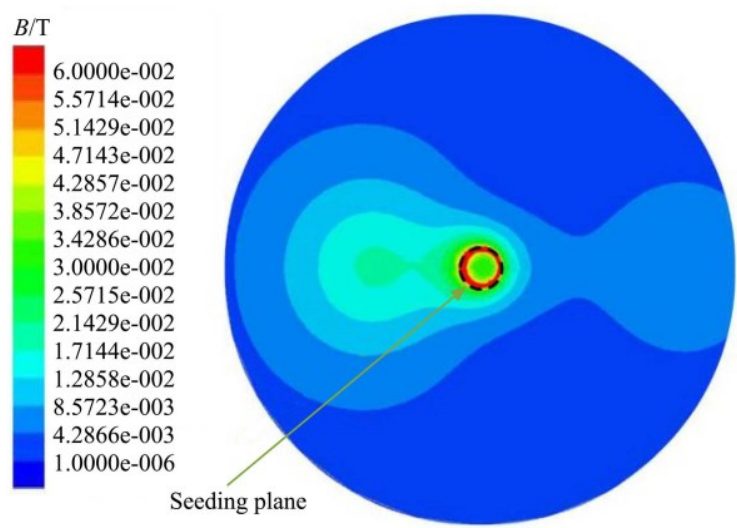

b. On the seeding plane

Figure 5 Magnetic flux density distribution when the CMS component works in the clearing state

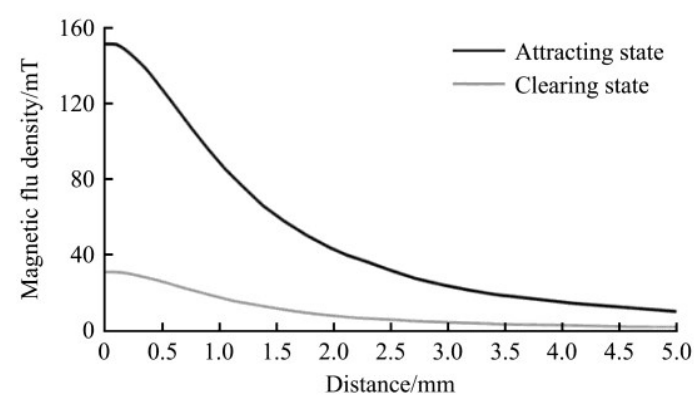

a. Along the center line of the magnetic head

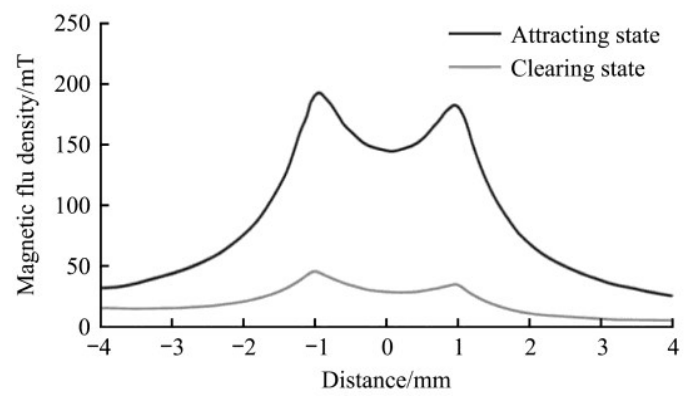

b. On the radius direction of the seeding plane

Note: Along the permanent-magnet II is taken as the positive direction.

Figure 6 Magnitude of the magnetic flux density

Figure $6 \mathrm{~b}$ illustrates the magnitude of the magnetic flux density along the radius direction of the seeding plane. In the Figure $6 \mathrm{~b}$, the peak values did not appear at the center point of the seeding plane but at the edges of the magnetic head (the radius of the magnetic head was $1 \mathrm{~mm}$ ). The peak values were $195.57 \mathrm{mT}$ and $181.77 \mathrm{mT}$ at the sides of permanent-magnet $\mathrm{I}$ and 
permanent-magnet II, respectively. The annular red area in Figure $5 \mathrm{~b}$ can also support the aforementioned rule that the edge of the seeding plane had higher magnetic flux densities compared to the inner area of the seeding plane. The magnitude of magnetic flux density was not symmetric along the radius direction. This may be due to the different lengths and diameters of two permanent-magnets. The maximum of the magnetic flux density in the clearing state was $47.25 \mathrm{mT}$ which was $24.16 \%$ of that in the attractive state.

According to the above results and analyses, we can conclude that in front of the seeding plane, the magnetic flux density of a cylindrical space, whose diameter and length were $2 \mathrm{~mm}$ and $3 \mathrm{~mm}$, had an obvious difference in the two working states.

However, in the attractive state, the magnetic field around each CMS component was so strong that the magnetic fields of other CMS components might be affected. This may, therefore, result in reseedings and miss-seedings. Therefore, interactions between any two adjacent CMS components need to be investigated. In order to reveal the spatial magnetic field distribution of these CMS components, the whole magnetic seed-metering device should be simulated in the Maxwell software.

\section{Spatial magnetic field distribution of the CMS magnetic seed-metering device}

3.1 Structure and workflow of the developed magnetic seed-metering device

The magnetic seed-metering device consisted of a roller and four rows of CMS components. The center angle of two adjacent rows of the CMS components was $90^{\circ}$ (Figure 7a). Moreover, the developed magnetic seed-metering device and its supporting facilities, such as a conveyor belt, a hopper, a vibrator, aluminum profiles and so on, comprised of a typical magnetic plug tray seeder, which is displayed in Figure 7a.

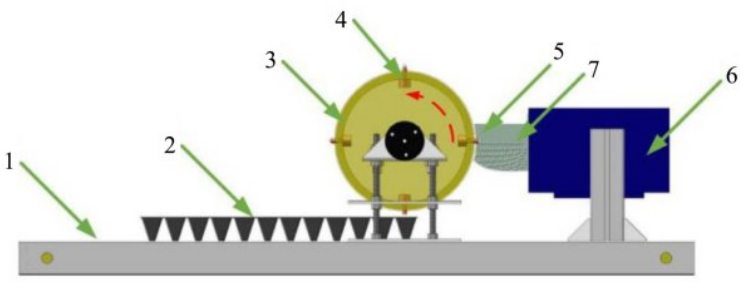

a. Typical magnetic precision plug tray seeder

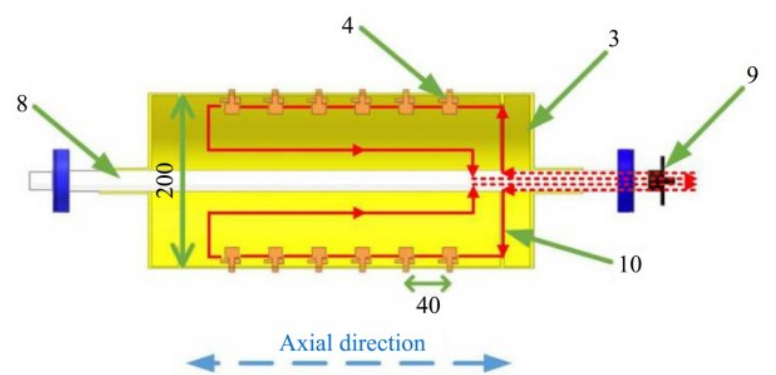

b. Cutaway view of the magnetic seed-metering device

1. Conveyor belt 2. Plug tray 3. Magnetic seed-metering device 4. CMS component 5. Hopper 6. Vibrator 7. Pelleted seed 8. Driven shaft 9. Collector ring 10 Wire

Figure 7 Structure of a magnetic precision plug tray seeder and the developed magnetic seed-metering device

The layout of CMS components in the roller with $200 \mathrm{~mm}$ is shown in Figure $7 \mathrm{~b}$. Along the axial direction, the interval between two adjacent CMS components was $40 \mathrm{~mm}$, which was as same as the distance between two adjacent plug tray cells of a universal plug tray. The coils of CMS components of each row were connected in series and connected with an external commutator via a collector ring.

The working flows of the developed magnetic seed-metering device were described as follows: initially, the CMS component was applied to the forward exciting current. After that, it worked in an attractive state. When a row of CMS components was passed by the hopper edgeways, it would pick pelleted seeds up. These pelleted seeds were carried until approaching the discharging position which was perpendicular to a row of plug tray cells without seeds. Then, this row of CMS components was applied reverse exciting current and switched to the clearing state. Therefore, the magnetic force generated by these CMS components disappeared and the pelleted seeds would fall into their corresponding plug tray cells.

After a delay, these CMS components were applied forward exciting current again. The next row of CMS components was rotated to the discharging position and meanwhile, the plug tray moved a distance as same as the length of a plug tray cell.

\subsection{Simulation model of the developed magnetic seed-metering device}

The developed magnetic seed-metering device can be seen as a spatial magnetic system. In order to study the interactions between these CMS components, a simplified model of the developed magnetic seed-metering device was built (Figure 8). Four rows of CMS components were averagely arranged in a cylindrical space whose diameter was as same as the roller.

In order to quantitatively describe the spatial magnetic field distribution of the developed seed-metering device, two auxiliary lines were added (Figure 8). The auxiliary line I was parallel to the axis direction of the cylindrical space. Since the diameter range of a pelleted seed was generally from $3 \mathrm{~mm}$ to $4 \mathrm{~mm}$, to reveal the magnetic field distribution at the center plane of a pelleted seed, the auxiliary line I was set $1.5 \mathrm{~mm}$ away from the seeding planes of CMS components. Besides, auxiliary line II passed the space points that were $1.5 \mathrm{~mm}$ away from the center points of four seeding planes on the periphery of the cylindrical space.

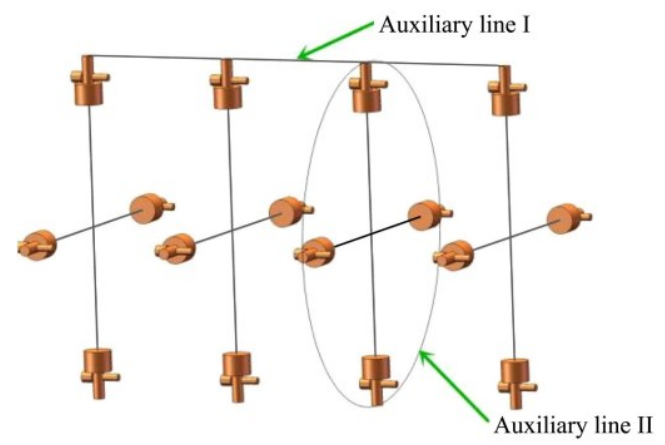

Figure 8 Simplified model of the developed magnetic seed-metering device

\subsection{Spatial magnetic field distribution of the developed magnetic seed-metering device}

Figure 9 illustrates the magnitudes of magnetic flux densities along the axial direction of the roller (i.e., auxiliary line I in Figure 8). The $x$-axis coordinate value of each spike corresponded to the center point of each seeding plane. As the analysis from Figure 6, the magnetic flux density would decrease gradually from the seeding plane. In the middle of two adjacent seeding components, the least magnetic flux density was $2.45 \mathrm{mT}$. It implicated that in the axial direction, the interaction between two adjacent seeding components was weak. Moreover, the peak values of magnetic 
flux densities at the centers of the four seeding planes were $61.1 \mathrm{mT}, 59.7 \mathrm{mT}, 63.5 \mathrm{mT}, 61.5 \mathrm{mT}$, respectively. This demonstrated that in the axial direction of the roller, the magnetic field distribution of each CMS components would not be affected by those of other CMS components, vice versa.

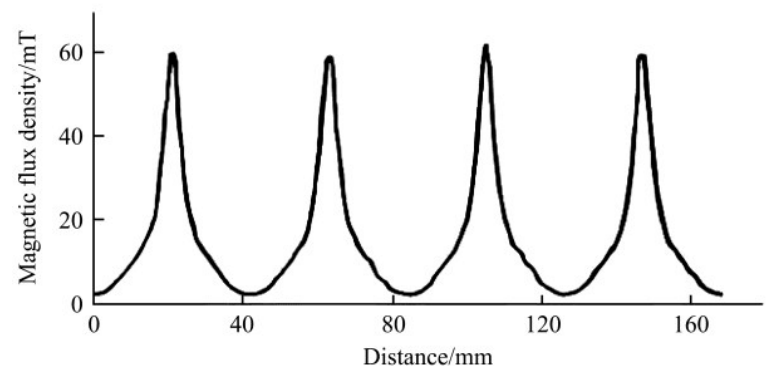

Figure 9 Magnitude of magnetic flux density along the center axis of the roller (the auxiliary line I in Figure 8)

Figure 10 shows the magnitudes of the magnetic flux densities on the peripheral direction of the roller (i.e., auxiliary line II in Figure 8). The $x$-axis coordinate values of these four spikes corresponded to the centers of the seeding planes along the perimeter of the roller. The magnetic flux density of the transitional section between the two CMS components was $0 \mathrm{mT}$. Therefore, pelleted seeds cannot be attracted by the surface of the roller.

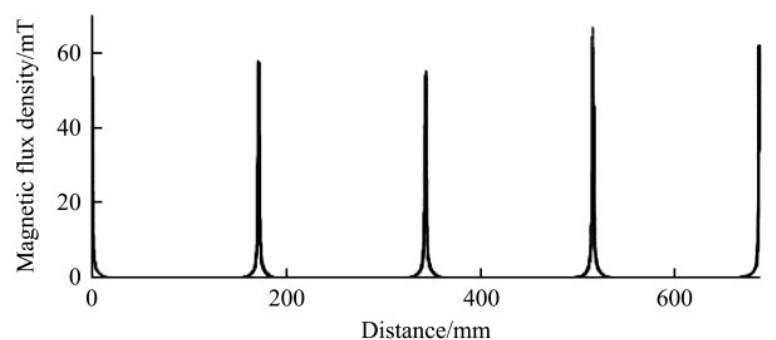

Figure 10 Magnitude of magnetic flux density along the peripheral direction of the roller (i.e., auxiliary line II in Figure 8)

According to the above analyses, unlike the electromagnetic seed-metering device ${ }^{[22]}$, the interactions between any two CMS components can be ignored. Moreover, the area of high magnetic flux density is concentrated on the seeding plane of each CMS seeding component. Hence, it was validated that the concentration ratio of the magnetic energy of the developed magnetic seed-metering device was higher than that of an electromagnetic seed-metering device. In both axial and circumferential directions of the roller, the magnitude of the magnetic flux density of each CMS component would not vary due to the inference caused by other CMS components. Furthermore, the magnetic flux density distribution of an individual CMS component indicated that the magnetic flux density on the seeding planes was different in the attractive state and the clearing one. These two important conclusions suggested that the seed-metering device can achieve a desirable single rate if the operational parameters are appropriate. In order to validate whether the practical seeding performance would be as same as the above-analyzed situation, a seeding performance experiment was conducted when the exciting current and the rotational speeds of the roller were in the common value ranges.

\section{Seeding performance experiment}

\subsection{Experimental factors and evaluation criteria}

A prototype experiment was conducted to validate the practical seeding performance of the developed magnetic seed-metering device. Figure 11 shows the prototype of the developed magnetic plug tray seeder.

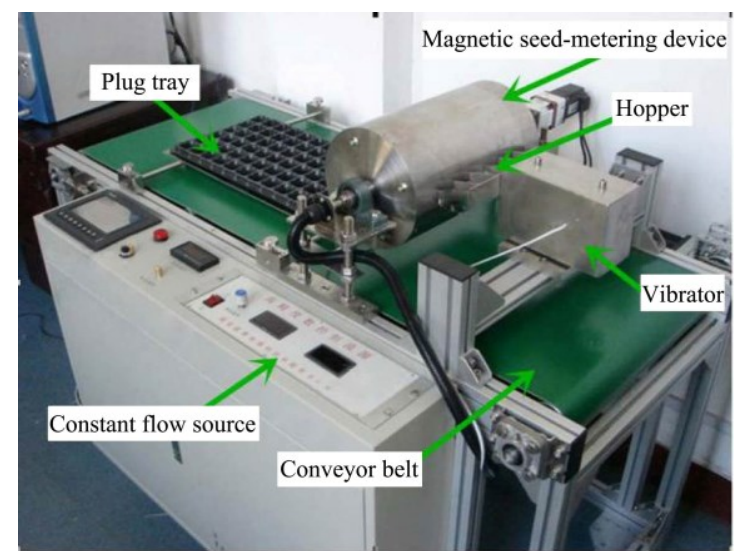

Figure 11 Prototype of the developed magnetic plug tray seeder

According to the outcomes of previous researches ${ }^{[17,22]}$, the rotational speed of the roller $n$ and the exciting current of coil $i$ were viewed as the factors of the seeding performance. Rape seeds were sown in the seeding performance experiment. They were pelleted and covered by the coating powder (containing 15\% iron powder) before the experiment. The physical characteristics of pelleted rape seeds are listed in Table 5 .

Table 5 Physical characteristics of the experimental pelleted rapeseed

\begin{tabular}{lccc}
\hline Seed type & Thousand seed weight $/ \mathrm{g}$ & Average diameter $/ \mathrm{mm}$ & Shape \\
\hline Rapeseed & 40.30 & 3.679 & Sphere \\
\hline
\end{tabular}

The developed magnetic precision seeder sowed the pelleted rape seeds under different rotational speeds $n$ and exciting currents $i$. A desirable seeding efficiency was 200 trays per hour. If the numbers of rows and columns of a plug tray were 12 and 6 , respectively, the rotational speed of the roller $n$ must be greater than $10 \mathrm{r} / \mathrm{min}$. Hence, the range of $n$ was set between $15 \mathrm{r} / \mathrm{min}$ to $30 \mathrm{r} / \mathrm{min}$. As for the exciting current $i$, the typical value was $0.2 \mathrm{~A}$ and its range was set from $0.125 \mathrm{~A}$ to $0.250 \mathrm{~A}$. The detailed levels of each factor are listed in Table 6 . The full factorial method was applied in the seeding performance experiment. Every experiment was repeated twice and 255 plug tray cells were seeded totally in each experiment.

Table 6 Experimental factors and levels

\begin{tabular}{lcccccc}
\hline \multicolumn{1}{c}{ Level } & 1 & 2 & 3 & 4 & 5 & 6 \\
\hline Rotational speed $n / \mathrm{r} \cdot \mathrm{min}^{-1}$ & 15 & 18 & 21 & 24 & 27 & 30 \\
Exciting current $i / \mathrm{A}$ & 0.125 & 0.150 & 0.175 & 0.200 & 0.225 & 0.250 \\
\hline
\end{tabular}

The performance of a magnetic seed-metering device was evaluated by the single rate, reseeding rate, and miss-seeding rate. The single rate referred to the percentage of the number of cells with one seed in the total number of cells. Besides, the reseeding rate meant the percentage of the number of cells with more than one seed in the total number of cells. Moreover, the miss-seeding rate was the percentage of the number of cells with no seed in the total number of cells. The above criteria can be calculated using Equations (4)-(6):

$$
\begin{aligned}
& s=\frac{N_{s}}{225} \times 100 \% \\
& r=\frac{N_{r}}{225} \times 100 \%
\end{aligned}
$$




$$
m=\frac{N_{m}}{225} \times 100 \%
$$

where, $s$ is the single rate, $\% ; N_{s}$ is the number of cells with one seed; $r$ is the reseeding rate, $\% ; N_{r}$ is the number of cells with more than one seed; $m$ is the miss-seeding rate, $\% ; N_{m}$ is the number of cells with no seed.

\subsection{Resultant analysis of seeding performance experiment}

According to the results of the seeding performance experiment, the single, reseeding and miss-seeding rates of the developed magnetic seed-metering device are shown in Figures $12 \mathrm{a}-12 \mathrm{c}$, respectively.

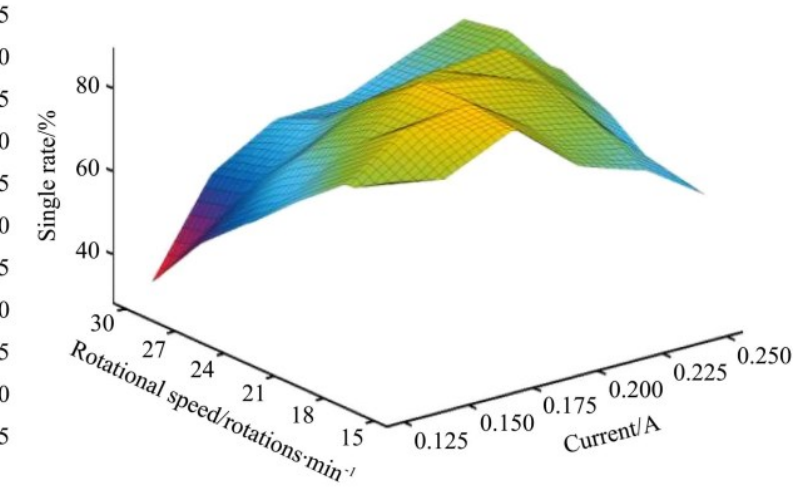

a. Single rate
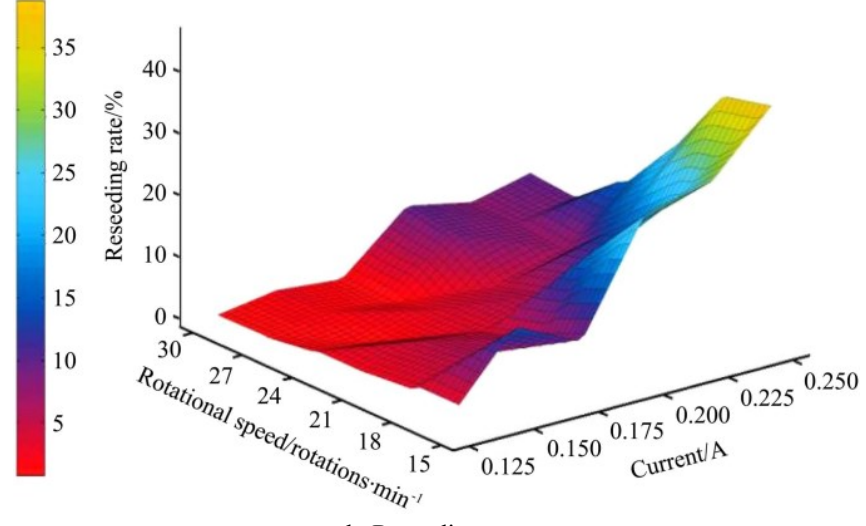

b. Reseeding rate
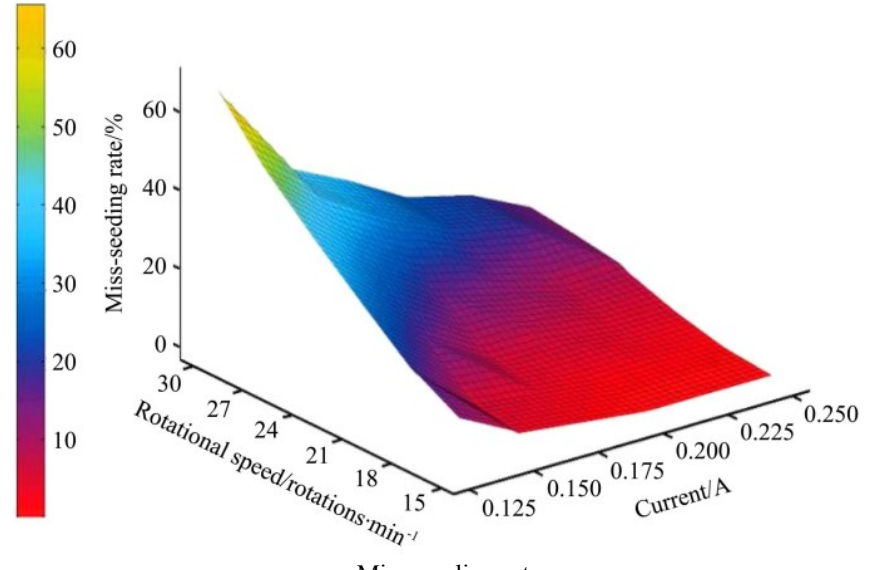

c. Miss-seeding rate

Figure 12 Effects of rotational speed $n$ and exciting current $i$ on seeding performance

If the exciting current $i$ maintained a certain value and the rotational speed $n$ rose gradually, the reseeding rate and miss-seeding rate would decrease and increase, respectively. As the rotational speed $n$ increased, the contacting time of a CMS component and pelleted seeds would decline. Therefore, more seeding components may not pick seeds up, resulting in miss-seedings. At a certain level of the rotational speed $n$, the reseeding rate and the miss-seeding rate would increase and drop as the exciting current $i$ rose. It can be validated by Equation (7) ${ }^{[16]}$,

$$
F_{m} \approx \frac{1}{2} \cdot(\alpha \cdot I \cdot N)^{2} \cdot \frac{\mu_{0} \cdot S_{0}}{\left(h_{0}\right)^{2}}
$$

where, $F_{m}$ is the magnetic force, $\mathrm{N} ; \alpha$ represents the magnetic descending coefficient, the range of $\alpha$ is from 0.45 to $0.85 ; S_{0}$ indicates the area of the seeding plane, $\mathrm{m}^{2} ; I$ is the exciting current, A; $N$ stands for the number of the coil windings; $h_{0}$ represents the air gap between the seeding plane and a pelleted seed at the moment of being attracted, $\mathrm{m}$.

As shown in Equation (7), the exciting current $i$ has a square relationship with the magnetic force. Hence, the greater the current is, the more likely reseeding.

In order to confirm whether the exciting current $i$ and the rotational speed $n$ significantly affect the seeding performance, one-way analyses of variance (ANOVA) between two factors and single, miss-seeding, reseeding rates were performed. The results of the one-way ANOVA are displayed in Table 7. The results in Table 7 show that both the exciting current $i$ and the rotational speed $n$ significantly influence the miss-seeding rate and reseeding rate ( $p$-value is less than 0.05 ), which was consistent with the previous analyses. However, neither of $n$ and $i$ had a significant influence on the single rate. It seems probable that as the exciting current $i$ rises, the miss-seeding rate decreased but the reseeding rate increased. Therefore, the sum of the miss-seeding rate and reseeding one was approximately unchanged. According to the above analyses, the exciting current $i$ cannot significantly affect the single rate. The same reason can be used to explain why the rotational speed $n$ could not affect the single rate either.

Compared to the pneumatic seed-metering device, the developed magnetic seeding component cannot be blocked and does not need replacing when sowing different seeds. However, its working efficiency is not as high as that of a pneumatic seed-metering device. The developed magnetic seed-metering device only can sow $200 \mathrm{plug}$ trays $/ \mathrm{h}$ to $300 \mathrm{plug}$ trays $/ \mathrm{h}$, while the pneumatic seed-metering device can sow more than 400 plug trays $/ \mathrm{h}$. Thus, the seeding efficiency of the developed magnetic seed-metering device needs improving.

As for the future direction, a kind of image processing software was developed by using a computer vision system to monitor miss-seeding and reseeding phenomena. If such problems happen, the corresponding plug tray cell positions could be marked. According to the specific problem of each marked cell, some auxiliary works, such as seeding again in the empty cells or clearing extra seeds in the reseeding cells, should be done subsequently.

In this experiment, the highest single rate was $90.2 \%$, when the rotational speed of roller $n$ was $21 \mathrm{r} / \mathrm{min}$ and the exciting current $i$ was $0.15 \mathrm{~A}$. Under this condition, the reseeding and miss-seeding rates were $3.92 \%$ and $5.88 \%$, respectively. The above results demonstrated that the seeding performance of the developed magnetic seed-metering device is acceptable under the optimal working parameters. 
Table 7 Results of analysis of variance between three seeding indicators

\begin{tabular}{|c|c|c|c|c|c|c|c|}
\hline Factor & Response & Variability & Sum of square & $d f$ & Mean square & $F$ & Significance \\
\hline \multirow{9}{*}{$\begin{array}{l}\text { Exciting } \\
\text { current } i\end{array}$} & \multirow{3}{*}{ Single rate $/ \%$} & Between groups & 1685.506 & 5 & 337.101 & 2.471 & 0.055 \\
\hline & & Within groups & 4092.63 & 30 & 136.421 & & \\
\hline & & Total & 5778.136 & 35 & & & \\
\hline & \multirow{3}{*}{ Miss-seeding rate $/ \%$} & Between groups & 1258.091 & 5 & 251.618 & 2.776 & 0.036 \\
\hline & & Within groups & 2719.654 & 30 & 90.655 & & \\
\hline & & Total & 3977.745 & 35 & & & \\
\hline & \multirow{3}{*}{ Reseeding rate $/ \%$} & Between groups & 4631.022 & 5 & 926.204 & 5.690 & 0.001 \\
\hline & & Within groups & 4883.353 & 30 & 162.778 & & \\
\hline & & Total & 9514.375 & 35 & & & \\
\hline \multirow{9}{*}{$\begin{array}{l}\text { Rotational } \\
\text { speed } n\end{array}$} & \multirow{3}{*}{ Single rate $/ \%$} & Between groups & 1429.287 & 5 & 285.857 & 1.972 & 0.112 \\
\hline & & Within groups & 4348.849 & 30 & 144.962 & & \\
\hline & & Total & 5778.136 & 35 & & & \\
\hline & \multirow{3}{*}{ Miss-seeding rate $/ \%$} & Between groups & 2064.821 & 5 & 412.964 & 6.476 & 0.000 \\
\hline & & Within groups & 1912.924 & 30 & 63.764 & & \\
\hline & & Total & 3977.745 & 35 & & & \\
\hline & \multirow{3}{*}{ Reseeding rate/\% } & Between groups & 4001.294 & 5 & 800.259 & 4.355 & 0.004 \\
\hline & & Within groups & 5513.081 & 30 & 183.769 & & \\
\hline & & Total & 9514.375 & 35 & & & \\
\hline
\end{tabular}

\section{Conclusions}

In this research, a CMS component was designed. The magnetic field distributions of the CMS component in both attractive and clearing states were obtained by FEM simulations. Based on the CMS components, a magnetic seed-metering device was developed. According to the results of the simulations and seeding performance experiment, four conclusions were obtained:

1) Pelleted seeds could be attracted and discharged successfully when the CMS component works in the attractive and clearing states because the magnitudes of magnetic flux density on the seeding plane in the attractive state were higher than that in the clearing state.

2) When the CMS component was applied an exciting current from $0.05 \mathrm{~A}$ to $0.30 \mathrm{~A}$, the maximum magnetic flux density was higher than that of the electromagnetic component. Moreover, the CMS component could adapt more types of pelleted seeds than the electromagnetic one, because the adjustable range of the magnetic flux density of the CMS component was wider than that of the electromagnetic component.

3) Pelleted seeds can be attracted to the seeding planes, rather than the surface of the roller. In the axial and radial directions of the roller, the magnetic flux densities between two adjacent CMS components were $2.45 \mathrm{mT}$ and $0 \mathrm{mT}$, respectively. The high magnetic flux densities only concentrated on the seeding planes of the CMS components and the magnetic field of each CMS component would not affect the others in both the axial and circumferential directions of the roller.

4) The reseeding rate would increase as the rotational speed $n$ decreased or the exciting current $i$ increased, while the miss-seeding rate would increase as the rotational speed $n$ increased or the exciting current $i$ decreased. The results of the seeding performance experiment show that when the rotational speed and current were $21 \mathrm{r} / \mathrm{min}$ and $0.15 \mathrm{~A}$. The single, reseeding and miss-seeding rates of the developed magnetic seed-metering device were $90.20 \%, 5.88 \%$, and $3.92 \%$, respectively. It shows that the seeding performance of the developed magnetic seed-metering device is acceptable, and thus the developed magnetic seed-metering device can be used in practical plug tray seeding processes.

In addition, the achievement obtained in this research could be used not only in agriculture but in other fields that need to discharge magnetic particles with uniform time intervals. For example, in the magnetic material processing field, this developed magnetic seed-metering device can put small magnetic particles into a chemical reactor quantificationally. Besides, the developed CMS component can replace conventional electromagnets in the solenoid valve since it can generate the same magnetic force using a lower exciting current.

\section{Acknowledgements}

This work was financially supported by the National Key Project of Research and Development Program, Ministry of Science and Technology of the People's Republic of China (Grant No.2016YFD020060102); Key Project of Research and Development Program (Agriculture) of Jiangsu Province (Grant No. E2017303). The authors acknowledge Saike Jiang from Jiangsu University for improving the quality of the artworks used in this work.

\section{[References]}

[1] Graamans L, Tenpierik M, Dobbelsteen A, Stanghellini C. Plant factories Reducing energy demand at high internal heat loads through facade design. Appl. Energy. 2020; 262: 114544. doi: 10.1016/j.apenergy.2020.114544.

[2] Mudarisov S, Badretdinov I, Rakhimov Z, Lukmanov R, Nurullin E. Numerical simulation of two-phase "Air-Seed" flow in the distribution system of the grain seeder. Comput Electron Agr, 2020; 168: 105151. doi: 10.1016/j.compag.2019.105151

[3] Jin X, Li Q W, Zhao K X, Zhao B, He Z T, Qiu Z M. Development and test of an electric precision seeder for small-size vegetable seeds. Int $\mathrm{J}$ Agric \& Biol Eng, 2019; 12(2): 75-81.

[4] Mao X, Yi S J, Tao G X, Yang L, Liu H Y, Ma Y C. Experimental study on seed-filling performance of maize bowl-tray precision seeder. Int $\mathrm{J}$ Agric \& Biol Eng, 2015; 8(2): 31-38.

[5] Ibrahim E J, Liao Q X, Wang L, Liao Y T, Yao L. Design and experiment of multi-row pneumatic precision metering device for rapeseed. Int J Agric \& Biol Eng, 2018; 11(5): 116-123.

[6] Zhang M H, Wang Z M, Luo X W, Zang Y, Yang W W, Xing H, et al Review of precision rice hill-drop drilling technology and machine for 
paddy. Int J Agric \& Biol Eng, 2018; 11(3): 1-11.

[7] Anantachar M, Kumar P G V, Guruswamy T. Neural network prediction of performance parameters of an inclined plate seed metering device and its reverse mapping for the determination of optimum design and operational parameters. Computers and Electronics in Agriculture, 2010; 72: 87-98.

[8] Cay A, Kocabiyik H, May S. Development of an electro-mechanic control system for seed-metering unit of single seed corn planters Part II: Field performance. Computers and Electronics in Agriculture, 2018; 145: $11-17$.

[9] Baral S S, Budhe V K, Kumara R, Swarnkar R. Development of tractor drawn electronic multi-crop planter cum fertilizer applicator for precision farming. International Journal of Advances in Agricultural Science and Technology, 2019; 6(10): 1-16.

[10] Gautam P V, Kushwaha H L, Kumar A, Kushwaha D K. Mechatronics application in precision sowing: A review. Int. J. Curr. Microbiol. App. Sci., 2019; 8(4): 1793-1807.

[11] Singh R C, Singh G, Saraswat D C. Optimisation of design and operational parameters of a pneumatic seed metering device for planting cottonseeds. Biosyst Eng, 2005; 92(4): 429-438.

[12] Ormond A, Furlani C, Oliveira M, Noronha R, Tavares T, Menezes P. Maize sowing speeds and seed-metering mechanisms. Journal of Agricultural Science, 2018; 10(9): 468-476

[13] Sriwongras P, Dostal P. Development of seeder for plug tray. Conference MendelNet, Czech, Listopadu, 2013; pp.867-871.

[14] Kim D E, Lee G I, Kang Y K, Kim Y H, Lee H J, Kim H H, et al.
Development of a semi-automatic seeder with vacuum nozzles of quadratic arrangement for small-sized seeds. J. of Biosystems Eng, 2011; 36(6): 434-443.

[15] Guarella P, Pellerano A, Pascuzzi S. Experimental and theoretical performance of a vacuum seeder nozzle for vegetable seeds. J. Agric. Engng Res., 1996; 64(1): 29-36.

[16] Hu J P, Mao H P. Analytical and experimental study on principle of precision seed-meter by magnetic force. Transactions of the CSAM, 2004 35(4): 55-58. (in Chinese)

[17] Li X Q. Open access publishing of scientific scholarly journals in China Master dissertation. Zhenjiang: Jiangsu University, 2006; 78p. (in Chinese)

[18] Hu J P, Wang Q R, Shao X P. Simulation on magnetic precision seed-metering device. Chin. Soc. Agr. Mach, 2010; 41(12): 35-38.

[19] Yan X Y, Hu J P, Lu C T, Wang X. Design and research on the control system of magnetic plate-type seeding production line. Adv. Mater. Res., 2013; pp.694-697.

[20] Wang Q R, Hu J P, Liu Q, Yan J C. Seeding element polarity arrangement on drum-type magnetic precision seeder. Computer and Computing Technologies in Agriculture III, 2010; 317: 455-460.

[21] Wang J, Hu J P, Wang Q R, Wang X. Simulation on magnetic field characteristics of permanent-magnet seed-metering device. Computer and Computing Technologies in Agriculture V, PT III, 2012; 370: 230-238.

[22] Zheng S N. Open access publishing of scientific scholarly journals in China. Master dissertation. Zhenjiang: Jiangsu University, 2009; 76p. (in Chinese) 